\title{
Concurrent Execution of Multiple NAS Parallel Programs on a Cluster
}

\author{
Adam K.L. Wong and Andrzej M. Goscinski \\ School of Information Technology, Deakin University, Geelong, Vic 3216, Australia \\ \{aklwong, ang\}@deakin.edu.au
}

\begin{abstract}
Currently, coordinated scheduling of multiple parallel applications across computers has been considered as the critical factor to achieve high execution performance. We claim in this report that the performance and costs of the execution of parallel applications could be improved if not only dedicated clusters but also non-dedicated clusters were used and several parallel applications were executed concurreontly. To support this claim we carried out experimental study into the performance of multiple NAS parallel programs executing concurrently on a non-dedicated cluster.
\end{abstract}

\section{Introduction}

Parallel processing has moved one step closer toward the computing mainstream by exploiting specialized dedicated clusters and MPI. These dedicated clusters are built using off-shelf elements (processors, memories and networks), which make them cost-effective computer systems.

The cost to performance ratio could be improved even further if non-dedicated clusters are used. These clusters are owned by many institutions, universities, business, and industry. They are made of not necessarily the fastest computers and networks, but still form a huge computational power that can be used to solve many problems that require parallel computing for high performance. Cluster's PCs in their working environments are on average idle for much more than $50 \%$ of time $[2,4,12]$. Therefore, a cluster has the potential of concurrently supporting a mixture of parallel and sequential applications of different users, which could lead to the improvement of the execution performance $[14,15,7]$. We claim that these computer systems can also be used cost-effectively to concurrently execute several parallel applications submitted by many competing users.

Parallel applications can share the computational resources of a cluster in two dimensions: space and time. Space-sharing is usually done by static allocation of processes to the available computers. Therefore, processes of different parallel applications can be mapped into different sets of computers of the cluster and the execution of those processes would not interfere with each other. On the other hand, time-sharing can be achieved if processes of parallel applications are mapped into the same computer and the local scheduler schedules these processes to share the CPU among them. 
The time-sharing approach can provide good response time and good throughput for applications in a multi-user and multi-programs environment. However, it has been considered that uncoordinated scheduling of processes from different applications would seriously hurt the performance of a cluster $[1,3,5,17,13]$ : the execution overhead can be up to over 15 times of the program execution time. To our knowledge, the only existing work which has shown an opposite result is [11]. Therefore, we believe that more experimental studies of this problem will provide not only a better understanding of the problem, lead industry, business and research institutions toward parallel processing on their existing clusters, but also form a background for the development of global scheduling facilities for computer clusters.

The aim of this paper is to confirm our claim by showing the results of our study into the performance of multiple parallel applications executing concurrently on a cluster. We also want to demonstrate that the results achieved by other researchers showing that uncoordinated scheduling of parallel applications would seriously hurt the performance of a cluster are unsubstantiated. For this purpose experiments were carried out using the well known and widely used NAS Parallel Benchmarks [9].

The paper is organized as follows. Section 2 presents the related work. Section 3 describes attributes of parallel applications required to carry out the proposed experiments on a cluster, and introduces the selected NAS benchmarks. Section 4 details the experiments carried out. Section 5 reports on the achieved results and presents the analysis of these results. Finally, the conclusion is presented in Section 6.

\section{Related Work}

Parallel computer systems such as the Massively Parallel Processors (MPPs) have a low communication overhead and therefore the effect of uncoordinated scheduling of processes from parallel applications executing concurrently on the systems is significant. Coordinated scheduling such as gang-scheduling [5] is normally used to alleviate the problem.

Time-sharing is intrinsically supported in a computer cluster via local scheduling. That means the local scheduler is responsible for time sharing of the CPU among all the processes which have been allocated to that computer. Processes from a parallel application can be placed into some or all of the computers in the cluster depending on the required parallelism. However, processes belonging to the same parallel application would not be guaranteed to execute at the same time across the computers in the cluster. Previous studies carried out using stimulation [1, 3, 17] have found that if the parallel application is communication intensive, uncoordinated scheduling of processes would lead to a great loss of performance because a process stalls when it communicates with a non-scheduled process.

$[13,11]$ present the results of co-scheduling of multiple parallel applications on a cluster using local scheduling, which are quite different. [13] shows that coscheduling of parallel applications on a cluster worsens their execution performance. However, that result is difficult to assess as the experiment is not clearly described and applications used in the experiment are not defined. [11] shows that local scheduling can out-perform gang-scheduling of parallel applications executing on a Beowulf cluster with a slow network (100Mbps Ethernet). 


\section{The NAS Parallel Benchmarks (NPB)}

We have selected to use the NAS Parallel Benchmarks (NPB) for this study because they have been widely used to objectively measure and compare the performance of parallel computer systems. In particular, the NAS Parallel Benchmarks 2.4 [16] can be used as parallel applications and run on non-dedicated clusters. Each NPB 2.x provides source codes written with MPI. Furthermore, these benchmarks can be coarsely divided into two major categories, computation-bound and communicationbound, which is important when carrying out experiments on commonly used clusters, with slow, 100Mbps, networks.

The NPB suite is a set of eight programs, which were derived from computational fluid dynamics (CFD) codes [9]. Each of these programs focuses on some important aspects of highly parallel supercomputing for aerophysics applications. There are two groups of these applications: five "kernels" and three "simulated computational fluid dynamics (CFD) applications". The kernels are relatively compact problems, easy to implement. They mimic the computational core of different numerical methods used by CFD applications - each of them emphasizes a particular type of numerical computation. They provide insight as to the general levels of performance that can be expected on these specific types of numerical computations. The simulated CFD applications are more difficult to implement - they indicate the types of actual data movement and computation required in state-of-the-art CFD application codes.

\subsection{Attributes of the Benchmark Programs Affecting the Scheduling Behaviour}

The behavior and scheduling study requirements led us to the specification of benchmark attributes that must be present because they influence the execution performance of a parallel application. These attributes form a basis of the selection of benchmarks for our experiments. They are as follows.

- Computation attributes: In general, the problem size of a parallel program is directly proportional to its execution time. Each of the programs of the NPB suite comes with several problem sizes (classes): A, B, C, W(orkstation) and S(ample). Excluding classes $\mathrm{W}$ and $\mathrm{S}$, class $\mathrm{A}$ is the smallest whereas class $\mathrm{C}$ is the largest.

- Communication attributes: Different parallel program has different communication features. The communication features of the NAS programs can be considered in two aspects: communication volume and communication pattern. In respect to the communication volume, the programs can broadly be classified into three categories: low, medium and high. In respect to the communication pattern, two forms exist: point-to-point and collective.

- Memory attributes: The size of main memory of a program during execution affects the scheduling behaviour, in particular could lead to memory swapping. The memory scheduling behavior of the NAS programs depends on the program size, characterized by the program class.

- Topology attributes: The software topology of processes of a parallel program defines the size (number of processes) and the structure (the connections of processes) of the program. The NAS programs use various software topologies. FT, MG, CG, LU and IS run well with a power-of-2 number of processes; SP and BT run well with a square number of processes; and EP runs with any number of process. 
Table 1. NAS programs with communication specific attributes

\begin{tabular}{|c|c|c|}
\hline Program Name & Communication Volume & Communication Pattern \\
\hline EP & Negligible & Negligible \\
\hline LU & Low & Point-to-Point $>>$ Collective \\
\hline BT & Medium & Point-to-Point $>>$ Collective \\
\hline MG & High & Point-to-Point $>$ Collective \\
\hline
\end{tabular}

\subsection{Selected NAS Benchmarks}

To evaluate the impact of concurrent execution of multiple parallel applications on their performance, we carried out an analysis of the NAS programs to identify those that posses the attributes addressed in Section 3.1. We have found that four programs, EP, LU, BT and MG, of the NBS suite, represent a broad range of communication patterns of parallel applications that can commonly be found in real world. The communication features of these programs are summarized based on $[6,9,10]$ and shown in Table 1.

We have chosen a problem size of class B for the programs listed above to make sure that (i) the execution time is long enough for the scheduling behaviour to be observed (only the number of iterations of execution in some programs have been altered), and (ii) memory swapping would not happen.

We could not satisfy fully the topology requirement. The reason is as follows. The MG and LU belong to the group of those programs that perform best (require) a power-of-2 number of processes, and EP can also be executed effectively with the same number of processes, BT performs best when it runs with a square number of processes, i.e., 1, 4, 9, 16. We assumed that the loss of performance for executing BT with eight (processes) rather than nine (processes) will not distort the experiment outcomes, and carried out the experiments using a power-of- 2 number of processes for each selected NAS program. Thus, the four programs, two kernel (EP and MG) and two simulated (LU and BT) applications, which posses the attributes to carry out our study, are selected.

\section{Experiments}

All of the scheduling experiments were carried out on a cluster which consists of 16 Pentium III computers, each with 383 Mbytes of main memory. The computers are connected together by a 100Mbps Fast Ethernet network. Each computer runs the Red Hat Linux operating system with the parallel programming support of MPI.

We decided to use a two level scheduling system, where the upper level is responsible for allocation of processes of each parallel application to cluster computers and the lower level schedules local parallel processes (of one or more than one application) running on each local computer of the cluster. MPI provides initial allocation of parallel processes to cluster computers. On an individual computer these processes are scheduled by the Red Hat Linux operating system scheduler.

An MPI application usually can be executed by first constructing a network topology, which specifies in the topology configuration file (TCF) the number and the identity of the computers used for the execution. Such a network topology can then be booted up in the cluster, which basically starts a MPI daemon process on each of the 
computers specified in the TCF. The MPI daemons are responsible for handling communications among processes of a parallel application. We used in our experiment the LAM/MPI implementation [8] as it is one of the most popular implementations of the MPI specification, and its version is LAM/MPI-6.5.9.

First, we measured the execution times of the four selected NAS parallel programs (EP, LU, MG and BT) individually. Taking into account the topology attributes specified in [9]: a power-of-2 of processes for LU and MG, a square number of processes for BT and any number of processes for EP, we selected a power-of-2 of processes as the standard topology for all the four programs executing in this experiment. Since there are 16 computers in our cluster, each of the programs were run on 2, 4, 8 and 16 computers; all of the programs were compiled for using 16 processes. Then, we measured i) the execution time for each of the selected NAS benchmarks when multiple copies of itself were executed concurrently on a cluster of 16 computers, and ii) the execution time for each of the selected NAS benchmarks when one or multiple copies of EP were executed concurrently with itself on the same cluster. The influence of the local scheduling was then observed and measured.

\section{Results and Analysis}

Speedup of NAS Programs. Our experimental study was carried out using the programs of Class B of NPB.

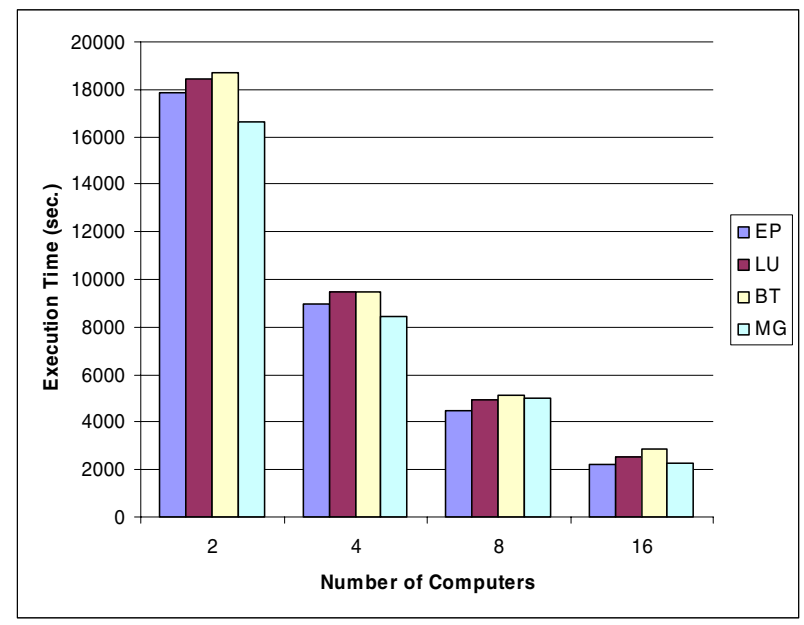

Fig. 1. Execution Time of NAS programs against different number of computers

Because the number of iterations of execution in the selected NAS parallel programs were increased, the execution time of each of parallel programs: EP, LU, BT and MG, against different number of computers: 2, 4, 8 and 16 of our cluster was measured. The result is presented in Figure 1.

The results we achieved are consistent with the benchmarking results listed in [9]. Besides, our results indicate that using a cluster of 16 Pentium III computers to execute each of the NAS parallel programs requires around 40 minutes of time. We 
believe that this time duration for an execution of a parallel program not only is realistic but also long enough for observing the scheduling behaviour when multiple copies of the programs are executed concurrently on a cluster.

Concurrent Execution of the NAS Benchmark Programs. The purpose of the experiment was to determine the effect of co-scheduling of multiple NAS programs on a cluster with particular attention paid to the execution performance of the programs. Table 2 shows the results of co-scheduling of two and three identical NAS parallel programs on our 16-computers cluster.

Table 2. Multi-program scheduling of parallel programs from the NBS

\begin{tabular}{|c|c|c|c|c|c|c|c|c|c|}
\hline \multirow{3}{*}{ Program } & \multirow{2}{*}{\multicolumn{3}{|c|}{$\begin{array}{c}\text { Sequential } \\
\text { Execution Time } \\
(\text { SET) (sec.) }\end{array}$}} & \multirow{2}{*}{\multicolumn{3}{|c|}{$\begin{array}{c}\text { Concurrent } \\
\text { Execution Time } \\
(\mathrm{CET})(\mathrm{sec} .) \\
\text { Number of copies }\end{array}$}} & \multicolumn{3}{|c|}{ Slowdown $=$ CET $/$ SET } \\
\hline & & & & & & & \multicolumn{3}{|c|}{ Number of copies } \\
\hline & 1 & 2 & 3 & 1 & 2 & 3 & 1 & 2 & 3 \\
\hline EP & 2240 & 4480 & 6720 & 2240 & 4497 & 6711 & 1.00 & 1.00 & 1.00 \\
\hline LU & 2522 & 5044 & 7566 & 2522 & 4942 & 7478 & 1.00 & 0.98 & 0.99 \\
\hline BT & 2859 & 5718 & 8577 & 2859 & 5046 & 7561 & 1.00 & 0.88 & 0.88 \\
\hline MG & 2279 & 4558 & 6837 & 2279 & 4550 & 6769 & 1.00 & 1.00 & 0.99 \\
\hline
\end{tabular}

Expecting to observe some worse performance, we used the term slowdown to refer to the slowing down of a parallel program when multiple copies of the same program are running concurrently on the same system. It is calculated by dividing the time (CET) of concurrent execution of multiple copies of a program on a multiprogramming system by the time (SET) of sequential execution of multiple copies of the program run on the same system. Therefore, a slowdown value of greater than 1 implies a slowing down of the execution, and worse performance.

Table 3 shows the results of co-scheduling of the NAS parallel programs: LU, BT and MG executing with one, two and three copies of EP. The reason for that is EP is a computation-bound parallel application while all others are communication-bound parallel applications. Mixing computation- and communication-bound applications together can generate a higher degree of randomness for the processes to communicate and synchronize each other.

As the number of copies of a NAS parallel program increases, the total memory required for the executions increases significantly, especially in the data-spaces. In both cases, the memory requirement for programs to execute is carefully controlled to make sure that no memory swapping would occur. Our results show that no slowdown is greater than 1 in all of the above scheduling cases which is different from the results presented in the majority of the current publications.

The results indicate that both computation-bound and communication-bound parallel applications are not sensitive to co-scheduling on a cluster with a slow network. From table 2, it can also be seen that the slowdown obtained in LU, BT and MG is slightly less than 1 which can be explained by the low CPU utilization of the programs. When multiple copies of those programs are scheduled together, a blocked process can be scheduled to keep the CPU as busy as possible. 
Table 3. Mixed multi-program scheduling of parallel programs from the NBS

\begin{tabular}{|c|c|c|c|c|c|c|c|c|c|}
\hline \multirow{3}{*}{ Program } & \multicolumn{3}{|c|}{$\begin{array}{c}\text { Sequential } \\
\text { Execution Time } \\
{[\text { SET] (sec.) }}\end{array}$} & \multicolumn{3}{|c|}{$\begin{array}{c}\text { Concurrent } \\
\text { Execution Time } \\
{[\mathrm{CET}] \text { (sec.) }}\end{array}$} & \multicolumn{3}{|c|}{ Slowdown $=$ CET $/$ SET } \\
\hline & \multicolumn{3}{|c|}{ Number of copies of EP } & \multicolumn{3}{|c|}{ Number of copies of EP } & \multicolumn{3}{|c|}{ Number of copies of EP } \\
\hline & 1 & 2 & 3 & 1 & 2 & 3 & 1 & 2 & 3 \\
\hline LU & 4762 & 7002 & 9242 & 4719 & 6951 & 9211 & 0.99 & 0.99 & 1.00 \\
\hline BT & 5099 & 7339 & 9579 & 4752 & 6964 & 9188 & 0.93 & 0.95 & 0.96 \\
\hline MG & 4519 & 6759 & 8999 & 4475 & 6705 & 8962 & 0.99 & 0.99 & 1.00 \\
\hline
\end{tabular}

\section{Conclusions}

In this paper we have presented the results of the experimental study into the performance of multiple parallel applications executing concurrently on a cluster. The aim was to confirm our claim that the performance of the execution of parallel applications could be improved when several parallel applications are executed concurrently, and to show that the claim made by other researchers that uncoordinated scheduling of parallel applications would seriously hurt the performance of a cluster is unsubstantiated.

Contrary to the results presented in most of the current literature, we have found that even if a parallel application is communication intensive, there is no performance loss of the parallel application due to uncoordinated communications and synchronizations of processes. Our study of the concurrent execution of multiple parallel applications on a cluster does not confirm the simulation results reported in $[1,3,17]$, which recommend synchronized scheduling of multiple parallel applications such as gang-scheduling. The results of our experimental study using real parallel applications are different from the result shown in [13]; the only result presented in the current literature which is inline with us is in [11].

We have demonstrated that concurrent execution of multiple parallel applications on a cluster did not make the execution performance of a parallel application worse. The execution performance was improved. It seems that this cost-effective scheduling scheme is particularly useful for computer cluster, especially with a slow network.

\section{References}

1. R.H. Arpaci, A.C. Dusseau, A.M. Vahdat, L.T. Liu, T.E. Anderson and D.A. Patterson. The Interaction of Parallel and Sequential Workloads on a Network of Workstations. In Proceedings of 1995 ACM Joint International Conference on Measurement and Modeling of Computing Systems, pages 267-278, May 1995.

2. A. Acharya, G. Edjlali and J. Saltz. The Utility of Exploiting Idle Workstations for Parallel Computation. In Proceedings of 1997 ACM Sigmetrics International Conference on Measurement and Modeling of Computer Systems, pages 225-236, May 1997. 
3. C. Anglano. A Comparative Evaluation of Implicit Coscheduling Strategies for Networks of Workstations. In Proceedings of 9th International Symposium on High Performance Distributed Computing (HPDC9), pages 221-228, August 2000.

4. W. Becker. Dynamic Balancing Complex Workload in Workstation Networks -- Challenge, Concepts and Experience. In Proceedings High Performance Computing and Networking (HPCN) Europe Lecture Notes on Computer Science (LNCS), pages 407-412, 1995.

5. D.G. Feitelson and L. Rudolph. Gang Scheduling Performance Benefits for Fine-Grained Synchronization. Journal of Parallel and Distributed Computing, 16(4):306-318, 1992.

6. A. Faraj and X. Yuan. Communication Characteristics in the NAS Parallel Benchmarks. In Proceedings of the 14th IASTED International Conference on Parallel and Distributed Computing and Systems (PDCS 2002), Nov. 2002.

7. A. M. Goscinski and A. K. L. Wong. Performance Evaluation of the Concurrent Execution of NAS Parallel Benchmarks with BYTE Sequential Benchmarks on a Cluster. Paper submitted to the $11^{\text {th }}$ International Conference on Parallel and Distributed Systems, ICPADSO5.

8. The LAM/MPI Homepage. URL: http://www.lam-mpi.org, lasted access: June 2004.

9. NAS Parallel Benchmarks. URL: http://www.nas.nasa.gov/Software/NPB/, last accessed: Nov. 2004.

10. J. Subhlok, S. Venkataramaiah and A. Singh. Characterizing NAS Benchmark Performance on Shared Heterogeneous Networks. In 11th International Heterogeneous Computing Workshop, April 2002.

11. P. Strazdins and J. Uhlmann. Local Scheduling out-performs Gang Scheduling on a Beowulf Cluster. Technical Report TR-CS-04-01, The Australian National University, 2004.

12. F. Tandiary, S.C. Kothari, A. Dixit and E. W. Anderson. Batrun: Utilizing Idle Workstations for Large-scale Computing. IEEE Parallel and Distributed Technology, 4(2):41-48, 1996.

13. F.C. Wong, A.C. Arpaci-Dusseau and D.E. Culler. Building MPI for Multi-Programming Systems using Implicit Information. In Proceedings of the 6th European PVM/MPI User's Group Meeting, pages 215-222, 1999.

14. A. K. L. Wong and A. M. Goscinski. Scheduling of a Parallel Computation-Bound Application and Sequential Applications Executing Concurrently on a Cluster - A Case Study. In Proceedings of the $2^{\text {rd }}$ International Symposium on Parallel and Distributed Processing and Applications (ISPA04), Dec. 2004.

15. A.K.L. Wong and A.M. Goscinski. The Performance of a Parallel Communication-Bound Application and Sequential Applications Executing Concurrently on a Cluster - A Case Study. In Proceeding of the $12^{\text {th }}$ International Conference on Advanced Computing and Communication (ADCOM-2004), Dec. 2004.

16. Rob F. Van der Wijngaart. The NAS Parallel Benchmarks 2.4 NAS Technical Report NAS-95-020, NASA Ames Research Center, Moffett Field, CA, 1995.

17. B.B. Zhou, X. Qu and R.P. Brent. Effective Scheduling in a Mixed Parallel and Sequential Computing Environment. In Proceedings of the 6th Euromicro Workshop of Parallel and Distributed Processing, pages 32-37, Jan. 1998. 